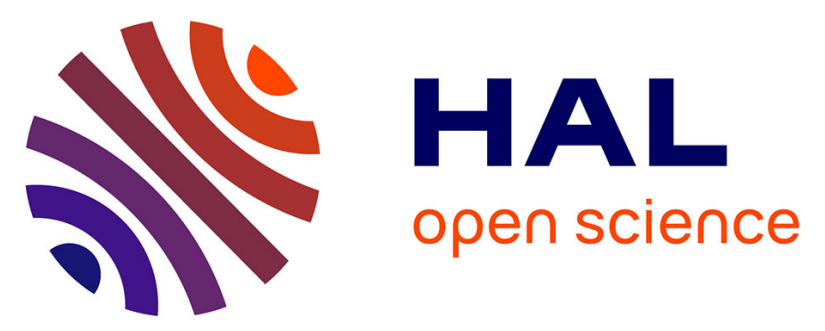

\title{
Preclinical Evaluation of 68Ga-DOTA-NT-20.3: A Promising PET Imaging Probe To Discriminate Human Pancreatic Ductal Adenocarcinoma from Pancreatitis
}

Aurélie Prignon, Claire Provost, Faisal Alshoukr, Dominique Wendum, Anne Couvelard, Jacques Barbet, Patricia Forgez, Jean-Noël Talbot, Anne

Gruaz-Guyon

\section{To cite this version:}

Aurélie Prignon, Claire Provost, Faisal Alshoukr, Dominique Wendum, Anne Couvelard, et al.. Preclinical Evaluation of 68Ga-DOTA-NT-20.3: A Promising PET Imaging Probe To Discriminate Human Pancreatic Ductal Adenocarcinoma from Pancreatitis. Molecular Pharmaceutics, 2019, 16 (6), pp.2776 - 2784. 10.1021/acs.molpharmaceut.9b00283 . hal-03046483

\section{HAL Id: hal-03046483 \\ https://hal.sorbonne-universite.fr/hal-03046483}

Submitted on 15 Dec 2021

HAL is a multi-disciplinary open access archive for the deposit and dissemination of scientific research documents, whether they are published or not. The documents may come from teaching and research institutions in France or abroad, or from public or private research centers.
L'archive ouverte pluridisciplinaire HAL, est destinée au dépôt et à la diffusion de documents scientifiques de niveau recherche, publiés ou non, émanant des établissements d'enseignement et de recherche français ou étrangers, des laboratoires publics ou privés. 


\title{
Preclinical Evaluation of ${ }^{68} \mathrm{Ga}-\mathrm{DOTA}-\mathrm{NT}-20.3$ : A Promising PET Imaging Probe To Discriminate Human Pancreatic Ductal Adenocarcinoma from Pancreatitis
}

\author{
Aurélie Prignon, ${ }^{* \dagger}$ ¿ Claire Provost, ${ }^{\dagger}$ Faisal Alshoukr, Dominique Wendum, ${ }^{\S}$ Anne Couvelard, \\ Jacques Barbet, ${ }^{\perp}$ Patricia Forgez, ${ }^{\#}$ Jean-Noël Talbot, ${ }^{\dagger, \text { qL }}$ and Anne Gruaz-Guyon \\ †Sorbonne Université, UMS28 Phénotypage du Petit Animal, Laboratoire d’Imagerie Moléculaire Positonique (LIMP), Paris 75020, \\ France \\ *Nuclear Medicine Department, CHR de Metz-Thionville, Thionville 57100, France \\ ${ }^{\S}$ Sorbonne Université, Pathology Department Saint-Antoine Hospital AP-HP, Paris 75012, France \\ "University of Paris, Pathology Department Bichat Hospital AP-HP, Paris 75006, France \\ ${ }^{\perp}$ GIP Arronax, Saint-Herblain 44800, France \\ \# Inserm UMRS 1007, Paris Descartes University, Paris 75006, France \\ IISorbonne Université, Nuclear Medicine Department Tenon Hospital AP-HP, Paris 75020, France
}

Supporting Information

\begin{abstract}
Neurotensin receptor 1 (NTSR1) is overexpressed in human pancreatic ductal adenocarcinoma (PDAC). Specific noninvasive positron-emission tomography (PET) imaging probes may improve the diagnostic accuracy and the monitoring of therapy for patients with PDAC. Here, we report the use of the ${ }^{68} \mathrm{Ga}$-labeled neurotensin (NTS) analogue DOTA-NT-20.3 to image human PDAC in animal models and to discriminate tumors from pancreatitis. In addition to the preclinical study, two tissue microarray slides, constructed by small core biopsies (2-5) from standard paraffin-embedded tumor tissues, were used to confirm the

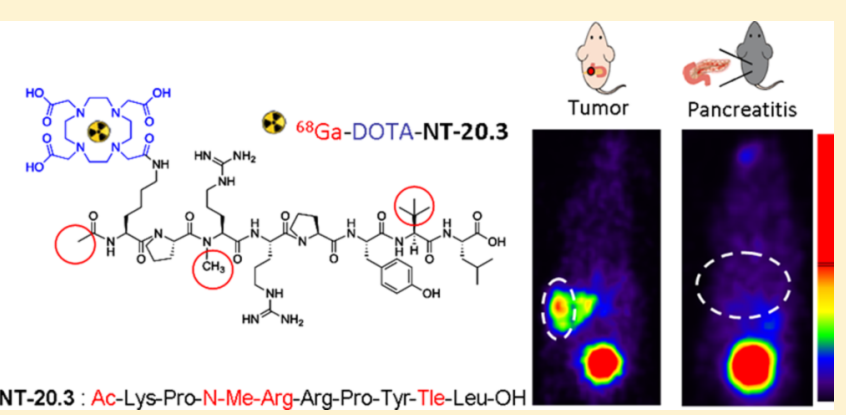
high $(78 \%)$ positivity rate of NTSR1 expression in human PDAC. PET imaging, biodistribution, blocking, and histology studies were performed in subcutaneous AsPC-1 pancreatic tumor-bearing mice. ${ }^{68} \mathrm{Ga}$-DOTA-NT-20.3 PET images showed rapid tumor uptake and high contrast between the tumor and background with a fast blood clearance and a moderate accumulation in the kidneys. Ex vivo biodistribution showed low uptake in normal pancreas $(0.22 \% \mathrm{IA} / \mathrm{g})$ and in the remaining organs at $1 \mathrm{~h}$ postinjection, kidney retention $(5.38 \pm 0.54 \% \mathrm{IA} / \mathrm{g})$, and fast clearance from blood and confirmed high uptake in tumors $(5.28$ $\pm 0.93 \% \mathrm{IA} / \mathrm{g}$ ), leading to a tumor-to-blood ratio value of 6 at $1 \mathrm{~h}$ postinjection. The significant decrease of tumor uptake in a blocking study demonstrated the specificity of ${ }^{68}$ Ga-DOTA-N-T20.3 to target NTSR1 in vivo. PET imaging was also conducted in an orthotopic xenograft model that allows tumors to grow in their native microenvironment and in an experimental pancreatitis model generated by caerulein injections. As opposed to $2-\left[{ }^{18} \mathrm{~F}\right]$ fluoro-deoxyglucose, ${ }^{68} \mathrm{Ga}-\mathrm{DOTA}-\mathrm{NT}-20.3$ distinguishes PDAC from pancreatitis. Thus, ${ }^{68}$ Ga-DOTA-NT-20.3 is a promising PET imaging probe for imaging PDAC in humans.
\end{abstract}

KEYWORDS: positron emission tomography (PET) imaging, gallium-68, neurotensin receptor, pancreatic ductal adenocarcinoma

\section{INTRODUCTION}

Pancreatic ductal adenocarcinoma (PDAC) is one of the most lethal cancers. The 1-year survival rate is around $18 \%$ for all stages of the disease, falling to less than $8 \%$ at 5 years. The poor prognosis of PDAC is mainly the result of late diagnosis, fast progression, and marginal benefits of conventional chemotherapy and targeted therapy: ${ }^{1}$ at the time of diagnosis, only $20 \%$ of patients have a nonmetastatic tumor and are eligible for curative surgical resection.
Initial diagnosis and disease staging are commonly accomplished using computed tomography (CT), magnetic resonance imaging (MRI), and endoscopic ultrasonography. ${ }^{2}$ Although these imaging techniques provide excellent anatomic details, they may not detect small hepatic, peritoneal, and other

Received: March 8, 2019

Revised: April 15, 2019

Accepted: April 23, 2019

Published: April 23, 2019 
distant metastases. ${ }^{3}$ Furthermore, neither of these imaging methods can confidently distinguish between malignant and nonmalignant cysts, especially for smaller lesions. Therefore, there is a real need to improve the early detection and staging of PDAC. ${ }^{4}$

Positron-emission tomography coupled to CT (PET/CT) using 2-[ $\left[{ }^{18} \mathrm{~F}\right]$ fluoro-deoxyglucose $\left({ }^{18} \mathrm{~F}-\mathrm{FDG}\right)$ improves the diagnosis and the management of patients: it can effectively detect primary pancreatic tumors and hepatic metastases commonly missed by $\mathrm{CT}$ and $\mathrm{MRI}^{5}$ It was reported to distinguish benign from malignant intraductal papillary mucinous neoplasms (IPMNs) of the pancreas. ${ }^{6}$ Nevertheless, ${ }^{18} \mathrm{~F}-\mathrm{FDG}$ is not sensitive enough for characterizing small intrapancreatic lesions or detecting small-sized metastases particularly in lymph nodes. ${ }^{18} \mathrm{~F}$-FDG also lacks specificity in differentiating between PDAC and focal mass pancreatitis, an inflammatory disease which shows significant ${ }^{18} \mathrm{~F}-\mathrm{FDG}$ uptake. $^{7}$

One approach to improve functional imaging is to take advantage of the abnormal expression of membrane receptors in the early stage of PDAC and to use them as targets for imaging agents. ${ }^{8}$ Neurotensin (NTS), a 13-amino acid gastrointestinal (GI) peptide, regulates GI functions including stimulation of pancreatic and biliary secretion, colonic motility, and growth of normal intestinal mucosa and pancreas, and consequently contributes in the digestion of nutriments. NTS action is mediated by three receptors. Two are $G$ proteincoupled receptors: the high-affinity neurotensin receptor (NTSR1) and the low-affinity neurotensin receptor (NTSR2). NTSR3 is a single-transmembrane receptor localized mainly in the trans-Golgi network that acts as a sorting protein. ${ }^{9}$ The action of NTS on islet endocrine secretion is principally mediated via the formation of a complex between NTSR2 and NTSR3. ${ }^{10}$ Moreover, NTS is involved in the progression of various solid tumors and may play a role in growth regulation and progression of PDAC. ${ }^{11}$ Those effects are mainly associated with an overexpression of NTSR1. ${ }^{12,13}$ NTSR1 has been identified on several PDAC cell lines, in human PDAC tissues, with lower expression in chronic pancreatitis or normal pancreas. ${ }^{14,15}$ Therefore, overexpression of NTSR 1 could be considered as a biomarker to diagnose and localize PDAC using noninvasive, nuclear medicine imaging probes.

In previous studies, we developed radiolabeled NTSR1 ligands ${ }^{16}$ and showed promising preliminary PET imaging results with gallium-68 $\left({ }^{68} \mathrm{Ga}\right)$. Indeed, ${ }^{68} \mathrm{Ga}$-DOTA-NT-20.3 allowed the detection of very small human colon carcinoma HT29 tumors. ${ }^{17}$ In the present study, we investigated the potential usefulness of ${ }^{68} \mathrm{Ga}-\mathrm{DOTA}-\mathrm{NT}-20.3$ for PDAC imaging and for discriminating between tumor and inflammation.

First, NTSR1 expression was evaluated by immunohistochemistry (IHC) in tissue microarrays (TMAs) from a cohort of patients operated for PDAC. The results of this rapid, relatively inexpensive, and efficient technique were compared to the published literature data to confirm the level of NTSR1 expression in PDAC. Then, ${ }^{68} \mathrm{Ga}$-DOTA-NT-20.3 was used to image subcutaneous AsPC-1 xenografts in mice and to confirm the potential of this tracer, in an orthotopic xenograft model that allows tumors to grow in their native microenvironment. Finally, ${ }^{68}$ Ga-DOTA-NT-20.3 was compared to the standard ${ }^{18}$ F-FDG PET imaging agent in pancreatic tumors and in an experimental pancreatitis model to demonstrate its ability to distinguish PDAC from pancreatitis. Given the high rate of expression of NTSR1 in PDAC and the favorable imaging properties of this new PET tracer, it is finally proposed that future clinical assessment of ${ }^{68} \mathrm{Ga}$-DOTA-NT-20.3 for PDAC imaging is warranted.

\section{EXPERIMENTAL SECTION}

NTSR1 IHC. IHC on TMA was used to evaluate the expression of NTSR1 in resected PDAC of 50 patients who underwent surgery in Hôpital Beaujon (Clichy, France) between 2000 and 2004. Clinical parameters were the following: 26 women and 24 men, aged $61 \pm 9$ years, tumor size $32 \pm 14 \mathrm{~cm}$, patients were scored $\mathrm{pT} 1 \mathrm{~N} 0=1, \mathrm{pT} 1 \mathrm{~N} 1=1$, pT2N0 $=1$, pT2N1 $=2$, pT3N0 $=5$, and pT3N1 $=40$.

De-paraffinized tissue sections $(4 \mu \mathrm{m})$ were subjected to heat-induced epitope retrieval in citrate buffer $(\mathrm{pH}$ 6.0). The sections were labeled for the target proteins using the avidinbiotin-peroxidase complex method. The slides were incubated at room temperature for $1 \mathrm{~h}$ with anti-NTSR 1 antibody $(1 / 50$; goat polyclonal sc-7596, Santa Cruz, USA). The slides were then incubated with biotinylated anti-goat IgG, (Vector Laboratories, Inc.). The antigen-antibody complex was revealed with avidin-biotin-peroxidase complex, according to the manufacturer's instructions for diamino-benzidine staining (Vectastain ABC Kit, Vector Laboratories, Inc.). All the slides were counterstained with hematoxylin, then scored by a pathologist (DW or AC). The immunohistochemical staining was evaluated in a semiquantitative manner: the localization of the staining was noted (cytoplasmic, membranous, nuclear) and a score $(0-300)$ was calculated by multiplying the labeling intensity (negative scored as 0 , weak as 1 , moderate as 2 , and strong scored as 3 ) by the percentage of stained cells $(0-100 \%)$. De-paraffinized tumors or tissues sections from animal models $(4 \mu \mathrm{m})$ were subjected to the same protocol.

PET Imaging Agents. The DOTA-NT-20.3 peptide (AcLys (1,4,7,10-tetraazacyclododecane-1,4,7,10-tetraacetic acid)Pro-Me-Arg-Arg-Pro-Tyr-Tle-Leu-OH) was produced by piCHEM and kindly provided by Iason $\mathrm{GmbH}$, Graz, Austria. It was labeled with ${ }^{68} \mathrm{Ga}$ as previously reported. ${ }^{17}$ The details and quality control are presented in Figure S1. Industrially produced ${ }^{18} \mathrm{~F}$-FDG was obtained from the nuclear medicine department of Hôpital Tenon.

Animal Models. All animal experiments were carried out in compliance with the French laws relating to the conduct of animal experimentation. The subcutaneous tumors (group 1) were developed by injection of $4 \times 10^{6}$ cells of the human pancreatic cell line AsPC-1 (ATCC, Rockville, MD, USA) in a $1: 1$ mixture of matrigel ( $\mathrm{BD}$ Biosciences) in the right shoulder of male immunodeficient mice (nu/nu, Charles River, France) (5-6 weeks old). The orthotopic tumors (group 2) were developed by surgical injection of AsPC-1 cells into the head of the pancreas. ${ }^{18}$ The immunodeficient mice $(\mathrm{nu} / \mathrm{nu})$ were anesthetized with isoflurane and analgesic treatment with 0.1 mg carprofen (Rimadyl, Pfizer) was applied $1 \mathrm{~h}$ before and 24 $\mathrm{h}$ after surgery. A small $(<1 \mathrm{~cm})$ incision was made in the left abdominal flank, through which the spleen was pulled out, allowing access to the pancreas. AsPC- 1 cells $\left(5 \times 10^{6}\right)$ in 100 $\mu \mathrm{L}$ phosphate-buffered saline were injected into the head of the pancreas. After inoculation, the organs were gently pushed back in the abdomen and the wound was closed in one layer with wound clips. A caerulein-induced model of acute pancreatitis (group 3) was used as previously described. ${ }^{19}$ 


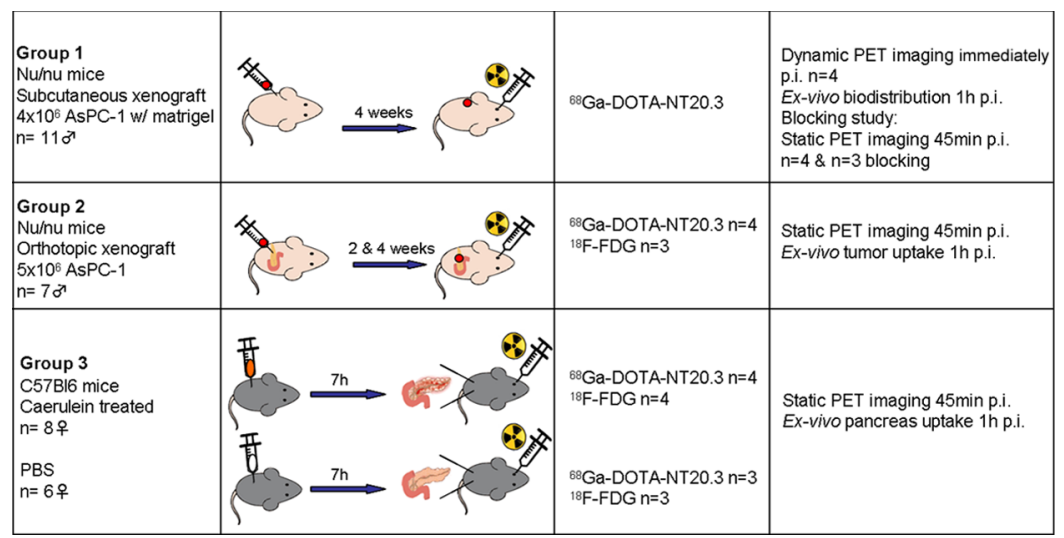

Figure 1. Experimental design.
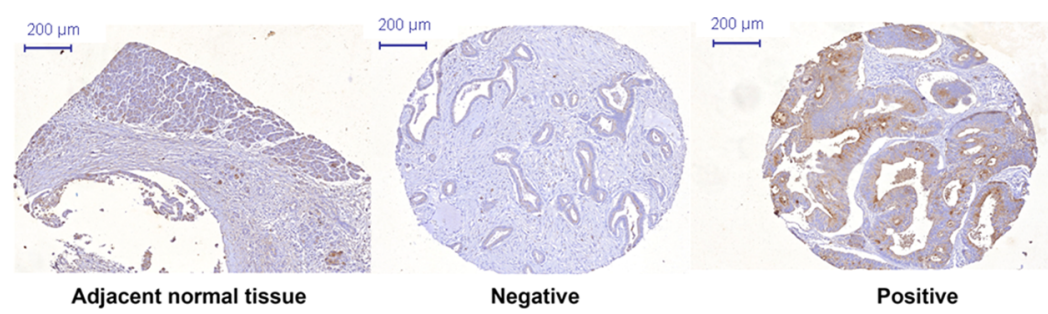

Figure 2. NTSR1 is highly expressed in PDAC. TMA analyzed with a Panoramic viewer at a $\times 20$ magnification; example of NTSR1 positive and negative staining in adenocarcinoma and a very weak staining in adjacent normal tissue.

Pancreatitis was induced in female C57Bl6 mice (5-10 weeks old) (Charles River, France) by administration of 7-hourly intraperitoneal injections of $50 \mu \mathrm{g} / \mathrm{kg}$ caerulein (Sigma, France). Control mice received comparable injections of 0.9 $\mathrm{mg} / \mathrm{mL}$ sodium chloride $(\mathrm{NaCl})$. The mice were sacrificed $7 \mathrm{~h}$ following the first injection of caerulein or saline during the acute phase. A schematic overview of the experimental design is presented in Figure 1.

PET Imaging and Analysis. Subcutaneous AsPC-1 tumorbearing mice (group 1, 4 weeks after cells' implantation) received an i.v. injection of 1 to $4 \mathrm{MBq}$ of ${ }^{68} \mathrm{Ga}-\mathrm{DOTA}-\mathrm{NT}$ 20.3 ( $\sim 00 \mathrm{pmol}$ ) via the retro-orbitary sinus. Using a smallanimal PET system (Mosaic, Philips Medical Systems, Cleveland, OH, USA), dynamic PET imaging over $50 \mathrm{~min}$ was performed immediately p.i. PET data were dynamically sorted into three-dimensional (3D) sinograms; the first series was $5 \times 1 \mathrm{~min}$ with $1 \mathrm{~min}$ between each sinogram and the second series was $4 \times 10 \mathrm{~min}$. All sinograms were reconstructed in $3 \mathrm{D}$ standardized uptake value (SUV) images and visualized as maximum intensity projection (MIP).

For all the following experiments, static PET images were acquired during an exposure time of $10 \mathrm{~min}, 45 \mathrm{~min}$ after injection of radiotracers. Other subcutaneous AsPC-1 tumorbearing mice from group 1 were submitted to check the specificity of the uptake (blocking study). Mice received $\sim 2.4$ MBq of ${ }^{68} \mathrm{Ga}-\mathrm{DOTA}-\mathrm{NT}-20.3(\sim 420 \mathrm{pmol})$, co-injected or not with $180 \mathrm{nmol}$ of nonlabeled NT-20.3 peptide, which represents more than 400 -fold excess of cold peptide.

Orthotopic AsPC-1 tumor-bearing mice from group 2 were imaged 2 and 4 weeks after tumor inoculation with ${ }^{68} \mathrm{Ga}$ DOTA-NT-20.3 or after a fasting period of $12 \mathrm{~h}$ with ${ }^{18} \mathrm{~F}-\mathrm{FDG}$. Every mouse received an injection of about $1.5 \mathrm{MBq}$. For the pancreatitis model (group 3), $6 \mathrm{~h} 45 \mathrm{~min}$ after the first injection of caerulein or saline, PET imaging was performed during the expected acute phase of pancreatitis, as described by
Lerch and Gorelick ${ }^{19}$ The mice received an injection of about $3 \mathrm{MBq}$ of ${ }^{18}$ F-FDG or ${ }^{68}$ Ga-DOTA-NT-20.3 (Figure 1).

Processing of reconstructed images was performed with the Syntegra-Philips software (PETView; Philips Medical Systems). For each mouse, 3D volumes of interest (VOIs) were drawn manually by irregular contouring on PET images as follows: total mouse, heart, kidneys, urinary bladder, tumor, and contralateral tumor-free background as a nontumor (NT) reference. Visual determination of VOIs was performed by consensus of two operators. The total number of voxels in a given organ was divided by the number of voxels in the "total mouse" for each frame. Data were expressed as percentage injected activity per organ (\% IA/organ) and the decaycorrected mean time-activity curves (TACs) were extracted for each target organ and tumor. Tracer uptake was quantified using maximal SUVs in the VOI $\left(\mathrm{SUV}_{\max }, \mathrm{g} / \mathrm{mL}\right)$ at each time point for tumor and NT and reported as the mean \pm standard deviation $(\mathrm{SD})$. The tumor-to-NT $(\mathrm{T} / \mathrm{NT})$ uptake ratios of $\mathrm{SUV}_{\max }$ were calculated.

Ex Vivo Biodistribution Studies. After imaging, $1 \mathrm{~h}$ p.i. of ${ }^{68}$ Ga-DOTA-NT-20.3, mice from group 1 were sacrificed and main organs and tissues were dissected, washed, weighed, and gamma-counted (1480 Wizard 3, PerkinElmer). Tumor or tissue uptake was expressed as mean \pm SD percentage injected activity/gram ( $\% \mathrm{IA} / \mathrm{g}$ ), corrected for radionuclide decay. In the blocking study, mice co-injected with ${ }^{68}$ Ga-DOTA-NT20.3 and unlabeled NT-20.3 (180 nmol) were also sacrificed 1 h p.i. and tumor uptake (\% IA/g) values were compared to those obtained in the absence of unlabeled NT-20.3 peptide.

Mice with orthotopic tumors (group 2, 4 weeks after tumor implantation) and mice with induced pancreatitis (group 3, $7 \mathrm{~h}$ following the first injection of caerulein or saline) were sacrificed $1 \mathrm{~h}$ p.i. of ${ }^{68} \mathrm{Ga}-\mathrm{DOTA}-\mathrm{NT}-20.3$ or ${ }^{18} \mathrm{~F}-\mathrm{FDG}$ injection. Tumor uptake (\% IA/g) was reported for each radiotracer. 


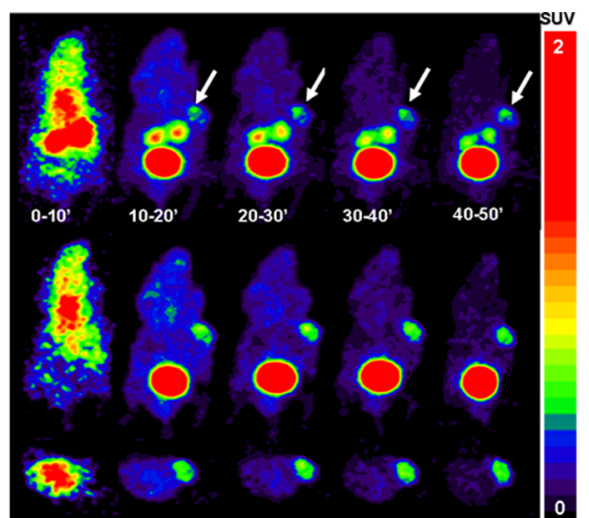

c

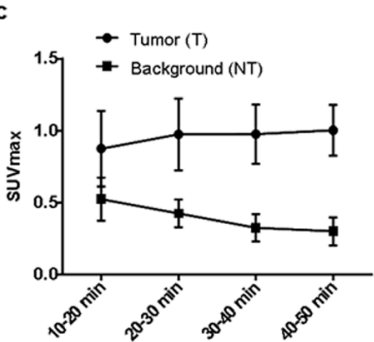

d

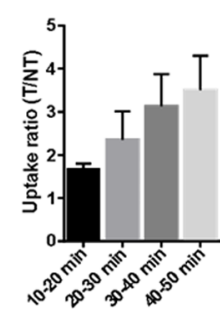

b
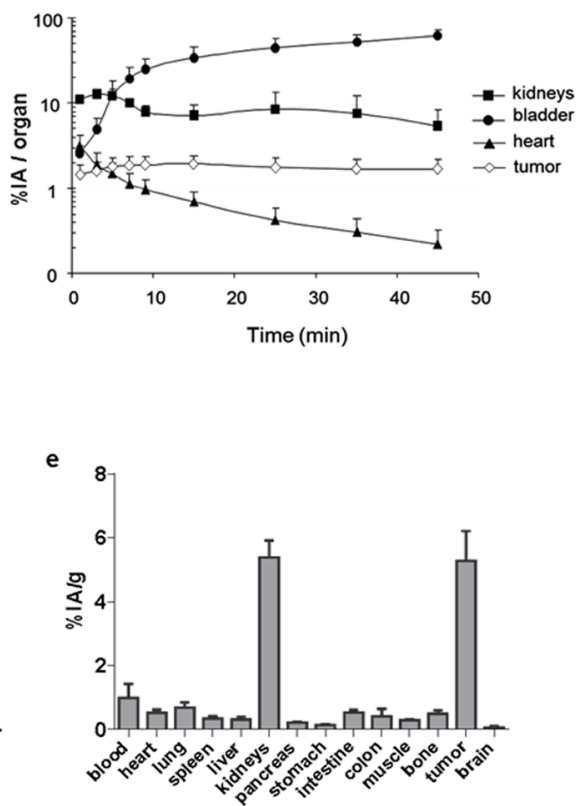

Figure 3. (a) Representative PET imaging of AsPC-1 tumor-bearing mice injected with $3 \pm 0.8 \mathrm{MBq}$; $\left(423 \pm 53\right.$ pmol) of ${ }^{68}$ Ga-DOTA-NT-20.3 ( $n$ = 4). Dynamic acquisition was started immediately p.i. over $50 \mathrm{~min}$. PET imaging was composed of five frames of 1 min computed in the same image and four frames of $10 \mathrm{~min}$. Upper image: MIP, middle image: coronal slice, lower image: transversal slice. Tumor uptake of ${ }^{68} \mathrm{Ga}-\mathrm{DOTA}-\mathrm{NT}$ 20.3 is clearly visible (arrow). (b) TAC analysis of dynamic PET imaging, data expressed in mean $\%$ of injected dose in the VOI (\% ID) \pm SD ( $n=$ 4). The data were obtained by quantitative analysis of dynamic PET images of AsPC-1 tumor-bearing mice at $0-50$ min p.i. of ${ }^{68} \mathrm{Ga}-\mathrm{DOTA}-\mathrm{NT}$ $20.3(n=4)$. (c) Time point of maximal SUV in tumor and background ROIs. Data expressed in mean SUV $\max _{ \pm} \pm$SD $(n=4)$. (d) PET imaging analysis in T/NT uptake ratio in mean $\pm \mathrm{SD}(n=4)$. (e) Ex vivo biodistribution of ${ }^{68} \mathrm{Ga}$-DOTA-NT-20.3 in AsPC-1 tumor-bearing nude mice at 1 $\mathrm{h}$ p.i. Tissue radioactivity is expressed as the percentage of injected activity per gram (\% IA/g, mean $\pm \mathrm{SD}$ ).

Statistical Analysis. Quantitative data are presented as mean $\pm S D$ and were compared using $t$-test for independent or for paired samples or using one-way analysis of variance followed by the Bonferroni test for multiple comparisons. Statistical analysis was performed using Prism 4.0 (GraphPad Software, La Jolla, CA). Level of significance was $P<0.05$.

\section{RESULTS}

NTSR1 Expression in Human PDAC. In cancer cells, NTSR1 labeling was intense, localized in the cytoplasm often with membrane reinforcement. All cells were labeled, some with very high intensity.

NTSR1 labeling was analyzed in TMA from 50 patients. Examples of positive and negative labeling in the TMA spot are shown in Figure 2. Labeling was scored according to the intensity and the percentage of labeled tumor cells; in nine patients $(18 \%)$ the specimens were considered strongly positive (membranous with a score between 201 and 300), in 19 patients $(38 \%)$ the specimens were scored positive (membranous with a score between 101 and 200), and in 11 patients $(22 \%)$ the tumor specimens had a weak (score between 0 and 100) but nevertheless membranous staining. The other specimens (20\%) had a weak cytoplasmic staining and one $(2 \%)$ was considered as negative. Altogether, $78 \%$ of PDAC overexpressed NTSR1.

PET Imaging and Biodistribution of ${ }^{68} \mathrm{Ga}-\mathrm{DOTA}-\mathrm{NT}-$ 20.3 in Mice Bearing Subcutaneous Human Pancreatic Adenocarcinoma. Dynamic PET imaging was performed 50 min after ${ }^{68} \mathrm{Ga}$-DOTA-NT-20.3 injection in $\mathrm{nu} / \mathrm{nu}$ mice with $300-500 \mathrm{~mm}^{3}$ subcutaneous AsPC-1 tumors (group 1). TACs were generated by VOI analysis. Four AsPC-1 tumor-bearing male mice received $3 \pm 0.8 \mathrm{MBq} ;(423 \pm 53 \mathrm{pmol})$ of ${ }^{68} \mathrm{Ga}-$ DOTA-NT-20.3. AsPC-1 tumors were clearly visible by PET as early as $10 \mathrm{~min}$ p.i. and easy to differentiate from NT tissue (Figure 3a). High-contrast PET images were obtained between 40 and 50 min p.i. $S U V_{\max }$ in tumors was $1 \pm 0.2$ with an NT background $\mathrm{SUV}_{\max }$ of $0.3 \pm 0.1$ (Figure 3c). Excretion in urine was fast, reaching $61 \%$ of IA in the bladder $50 \mathrm{~min}$ p.i. The accumulation of ${ }^{68}$ Ga-DOTA-NT-20.3 in the kidneys was moderate ( $5 \%$ of IA 45 min p.i.), reflecting good renal clearance of the activity. Tumor uptake plateaued over the PET acquisition period, representing $1.5 \%$ of $\mathrm{IA}$; it reached $1.7 \%$ of IA 45 min p.i. (Figure $3 \mathrm{~b}$ ). The clearance from blood was fast beginning at $3.1 \%$ of IA in the heart VOI p.i., decreasing to $0.2 \%$ of IA at 45 min p.i., resulting, as shown in Figure $3 \mathrm{~d}$, in $\mathrm{T} / \mathrm{NT}$ uptake ratios increasing from $1.7 \pm 0.1$ to $3.5 \pm 0.8$ between $10-20$ and $40-50 \mathrm{~min}$ p.i. No significant difference of radiotracer biodistribution was found between female and male mice (Figure S2).

Uptake of ${ }^{68}$ Ga-DOTA-NT-20.3 in major organs and blood investigated ex vivo $1 \mathrm{~h}$ p.i. by weighing and counting organs in a gamma counter confirmed the imaging results (Figure $3 \mathrm{e}$ ). A moderate uptake of ${ }^{68}$ Ga-DOTA-NT-20.3 (5.38 $\pm 0.54 \%$ IA/ g) was observed in kidneys. Very low tracer accumulation was observed in other organs, particularly in the pancreas $(0.22 \pm$ $0.03 \% \mathrm{IA} / \mathrm{g}$ ), consistent with the visual observations on PET imaging (Figure 3a). The circulating activity was $0.99 \pm 0.44 \%$ $\mathrm{IA} / \mathrm{g}$ and the uptake in AsPC-1 tumors was $5.28 \pm 0.93 \% \mathrm{IA} / \mathrm{g}$, corresponding to a tumor-to-blood ratio of $5.93 \pm 1.62$ (Figure $3 \mathrm{e})$. The tumor-to-muscle ratio was $18.21 \pm 4.01$ and the 
tumor-to-kidneys ratio was $1 \pm 0.26$. Table S1 (additional file) summarizes the data of the ex vivo biodistribution.

The specificity of tumor uptake of ${ }^{68}$ Ga-DOTA-NT-20.3 was demonstrated by co-administration of $180 \mathrm{nmol}$ (representing an over 400-fold excess) of unlabeled NT-20.3 (peptide with the same amino-acid sequence but without DOTA). SUV ${ }_{\max }$ in tumors was $1.8 \pm 0.6$ for the group of mice injected with ${ }^{68} \mathrm{Ga}$ DOTA-NT-20.3 alone versus $0.8 \pm 0.1$ for the blocking group corresponding to a $70 \%$ reduction of the PET uptake ratio $(\mathrm{T} /$ $\mathrm{NT}$ ) from $3.7 \pm 1.1$ to $1 \pm 0.1(P<0.005)$ (Figure $4 \mathrm{a}, \mathrm{b})$. The ex vivo determination of tumor uptake ( $1 \mathrm{~h}$ p.i.) was also reduced by $70 \%$ from $5.8 \pm 1.7$ to $1.6 \pm 0.5 \% \mathrm{LA} / \mathrm{g}(P<$ 0.005) (Figure 4c).

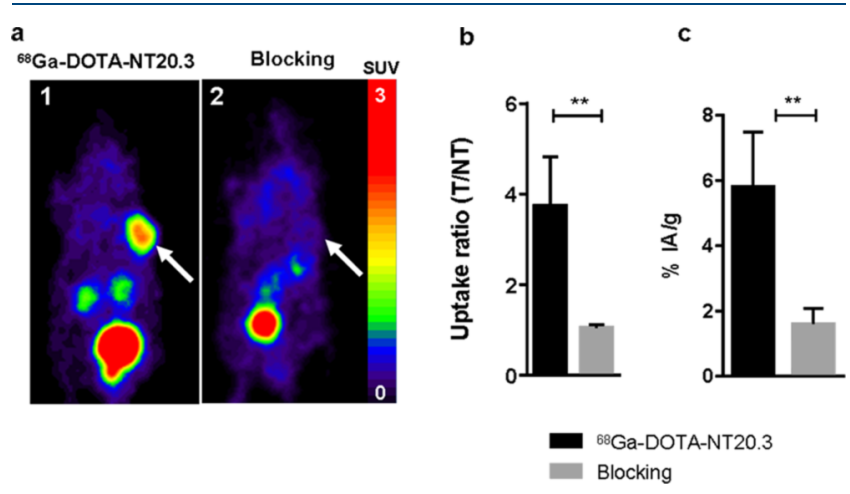

Figure 4. (a) Representative MIP PET imaging of blocking studies in AsPC-1 tumor-bearing mice. One mouse injected with $3.6 \mathrm{MBq}$ of ${ }^{68}$ Ga-DOTA-NT-20.3 (507 pmol; panel 1). One mouse co-injected with $2 \mathrm{MBq}(322 \mathrm{pmol})$ of ${ }^{68} \mathrm{Ga}-D O T A-N T-20.3$ and $180 \mathrm{nmol}$ of unlabeled NT-20.3 (panel 2). PET imaging was performed at 40-50 min p.i. (b) T/NT uptake ratio from PET imaging analysis. (c) Tumor radioactivity determined ex vivo ( $1 \mathrm{~h} \mathrm{p.i.)}$ and expressed as $\%$ $\mathrm{IA} / \mathrm{g}$. Data are given as mean $\pm \mathrm{SD}\left(n=4\right.$ for the ${ }^{68} \mathrm{Ga}$-DOTA-NT20.3 group and $n=3$ for the blocking group). $* * P<0.01$.

Comparative PET Imaging with ${ }^{68} \mathrm{Ga}-\mathrm{DOTA}-\mathrm{NT}-20.3$ and ${ }^{18} \mathrm{~F}-\mathrm{FDG}$ in Experimental Orthotopic PDAC. PET imaging was also performed in experimental orthotopic PDAC 45 min after ${ }^{68} \mathrm{Ga}-\mathrm{DOTA}-\mathrm{NT}-20.3$ or ${ }^{18} \mathrm{~F}-\mathrm{FDG}$ injection (Figure 5). Seven mice were imaged with ${ }^{68} \mathrm{Ga}-\mathrm{DOTA}-\mathrm{NT}$ $20.3(n=4)$ or with ${ }^{18}$ F-FDG $(n=3) 14$ and 30 days after cells' implantation. The mice were injected with ${ }^{68} \mathrm{Ga}$-DOTANT-20.3 (1.3 $\pm 0.3 \mathrm{MBq} ; 534 \pm 46 \mathrm{pmol})$ or, after a fasting period of $12 \mathrm{~h}$, with ${ }^{18} \mathrm{~F}$-FDG $(1.5 \pm 0.1 \mathrm{MBq})$.

A high background corresponding to uptake by normal tissues was observed with ${ }^{18} \mathrm{~F}-\mathrm{FDG}$ (Figure $5 \mathrm{~b}$ ) as compared to the very low background in normal organs, except for kidneys, obtained with ${ }^{68}$ Ga-DOTA-NT-20.3 (Figure 5a). Fourteen days after orthotopic implantation, the tumors were not palpable. PDAC was clearly detectable with ${ }^{68} \mathrm{Ga}$-DOTANT-20.3 because of the high contrast over the low background. This contrast was most pronounced at day 30 compared to day 14 (Figure 5a). In addition, the tumor uptake ratio was significantly higher for ${ }^{68}$ Ga-DOTA-NT-20.3 (4.6 \pm 1.5$)$ (Figure 5a,c), as compared to ${ }^{18} \mathrm{~F}-\mathrm{FDG}(1.2 \pm 0.1)$ (Figure $5 b, c)(* P<0.05)$. Ex vivo analysis confirmed these results, the tumor uptake being $7 \pm 1.4 \% \mathrm{IA} / \mathrm{g}$ versus $0.4 \pm 0.1 \% \mathrm{IA} / \mathrm{g}$ for ${ }^{68} \mathrm{Ga}$-DOTA-NT-20.3 and ${ }^{18}$ F-FDG, respectively.

After PET imaging, the expression of NTSR1 in tumors invading the pancreas was confirmed by IHC. NTSR1 labeling was frequent and intense (Figure $5 \mathrm{~d}$ panels 1 and 2). Some cells showed a very strong intracytoplasmic labeling dispersed or organized in a cluster. Other cells showed a discrete membrane labeling (Figure 5d panels 3 and 4).

Comparative PET Imaging with ${ }^{68} \mathrm{Ga}$-DOTA-NT-20.3 and ${ }^{18} \mathrm{~F}-\mathrm{FDG}$ in Experimental Pancreatitis. Female C57Bl6 mice received repeated intraperitoneal injections of supra-physiologic concentrations of caerulein $(50 \mu \mathrm{g} / \mathrm{kg})$, an analogue of cholecystokinin, to induce pancreatitis. ${ }^{20}$ The ${ }^{68}$ Ga-DOTA-NT-20.3 and the ${ }^{18}$ F-FDG uptake in pancreatic inflammation were compared. Seven animals were injected with $2.9 \pm 0.6 \mathrm{MBq}$ of ${ }^{68}$ Ga-DOTA-NT-20.3 (401 $\left.\pm 26 \mathrm{pmol}\right)$ and 7 mice were injected with $3.1 \pm 0.9 \mathrm{MBq}$ of ${ }^{18}$ F-FDG after a fasting period of $12 \mathrm{~h}$.

Figure $6 \mathrm{a}$ shows representative histological sections of the pancreas of treated and untreated mice. Cearulein administration caused pancreatic edema, infiltration by inflammatory cells between the acini and into the peripancreatic fat, and fibrosis. NTSR1 labeling was dispersed inside the cytoplasm in cearulein-treated and control animals. The labeling was weak and of the same intensity in treated mice with acute pancreatitis or in control mice. ${ }^{18} \mathrm{~F}-\mathrm{FDG}$ PET imaging confirmed the extent and intensity of pancreatic inflammation in situ. As shown in Figure $6 \mathrm{~b}$ image 1, in treated mice with acute pancreatitis, ${ }^{18}$ F-FDG uptake in the pancreas was elevated and confirmed by an ex vivo strong and significant uptake (11\% IA/g). By contrast, in control mice, only a low ${ }^{18}$ F-FDG accumulation was observed in the pancreas (Figure $6 \mathrm{~b}$ image 2$)$ with an ex vivo uptake of $4 \% \mathrm{IA} / \mathrm{g}(* * * P<$ 0.005). As expected, ${ }^{68}$ Ga-DOTA-NT-20.3 PET imaging did not show radiotracer uptake in any of the conditions (Figure 6 b images 3 and 4). ${ }^{68}$ Ga-DOTA-NT-20.3 uptake was weak and similar in pancreas of treated and untreated animals $(1.5$ and $2 \% \mathrm{IA} / \mathrm{g}$ ), respectively (Figure $6 \mathrm{c}, \mathrm{d}$ ). In pancreatitis, ${ }^{68} \mathrm{Ga}$ DOTA-NT-20.3 uptake was significantly weaker $(1.5 \% \mathrm{IA} / \mathrm{g})$ as compared to ${ }^{18} \mathrm{~F}-\mathrm{FDG}$ uptake $(* * * * P<0.001)$.

\section{DISCUSSION}

NTSRs have been described for the first time in 1998 by Reubi et al. as overexpressed in human PDAC. Using in vitro autoradiography with ${ }^{125} \mathrm{I}-\left[\mathrm{Tyr}^{3}\right]$-NTS, they found expression of membrane receptors in $75-88 \%$ of human PDAC. In contrast, NTSRs were not detected in normal human pancreas, in chronic pancreatitis, as well as in endocrine pancreatic tumors. ${ }^{14}$ These data were extended to liver metastases and in late-stage precursor lesions called pancreatic intraepithelial neoplasia (PanINs). ${ }^{21}$ Overexpression of NTSR1 was also demonstrated by IHC using rabbit antihuman NTSR1 in paraffin-embedded patient samples. NTSR1 expression was observed in $85 \%$ of PDAC 22 and more recently in $79.4 \%$ in a larger cohort of PDAC (104/131) and only in $22.7 \%(10 / 44)$ of pancreatitis $(P<0.001) .^{15}$

In addition to these conventional IHC analyses, we used two TMA slides, constructed by small core biopsies (2-5) from standard paraffin-embedded tumor tissues, allowing simultaneous analysis of tumor specimens from 50 patients in uniform experimental conditions. With this high-throughput technology and with a goat antihuman NTSR1, we confirmed a $78 \%$ positivity rate of NTSR1 expression. This result complements what has been reported in the literature and suggests that detection of NTSR1 expression by IHC on miniaturized histological samples coming from biopsy may be used to manage pancreatic cancer and to follow malignant trans- 
a

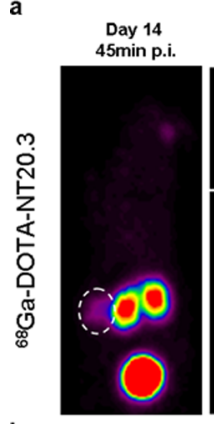

b

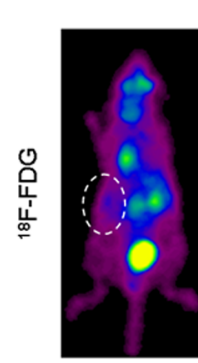

Day 30
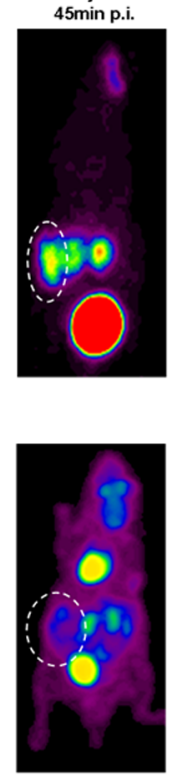

c
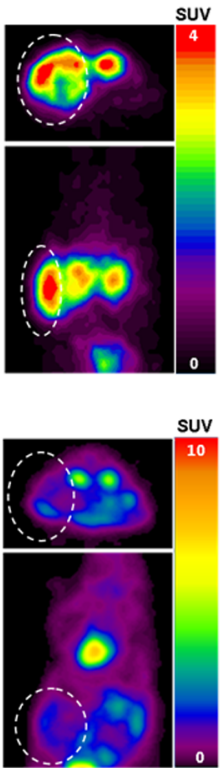

d
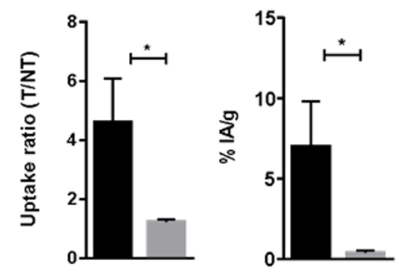

- ${ }^{68}$ Ga-DOTA-NT20.3

-18 F-FDG

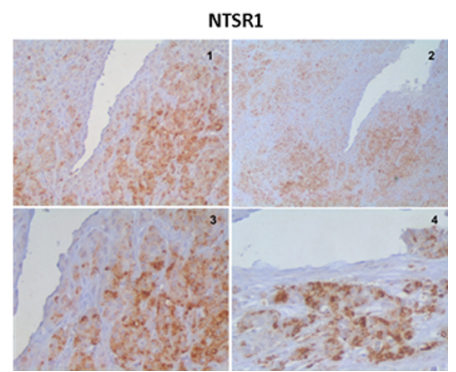

Figure 5. Comparative PET imaging 45 min p.i. of ${ }^{68}$ Ga-DOTA-NT-20.3 (a) and ${ }^{18}$ F-FDG (b) in an orthotopic PDAC model. Representative in vivo PET images of orthotopic AsPC-1 tumor-bearing mice 14 and 30 days after cells' implantation. MIP and transversal slices. Circles landmark the tumor. (c) T/NT uptake ratio from PET imaging analysis at day 30 and tumor radioactivity determined ex vivo $1 \mathrm{~h}$ p.i. after in vivo PET imaging and expressed as \% IA/g. The mice were injected with ${ }^{68} \mathrm{Ga}-\mathrm{DOTA}-\mathrm{NT}-20.3(1.3 \pm 0.3 \mathrm{MBq} ; 534 \pm 46$ pmol $)$ or with ${ }^{18} \mathrm{~F}-\mathrm{FDG}(1.5 \pm 0.1$ MBq). Data are expressed in mean \pm SD $\left(n=4\right.$ for the ${ }^{68}$ Ga-DOTA-NT-20.3, $n=3$ for the ${ }^{18}$ F-FDG $) * P<0.05$. (d) NTSR1 IHC of an orthotopic AsPC-1 tumor invading the pancreas. Magnification $\times 100$ (panel 1), $\times 40$ (panel 2), and $\times 200$ (panels 3 and 4 ).
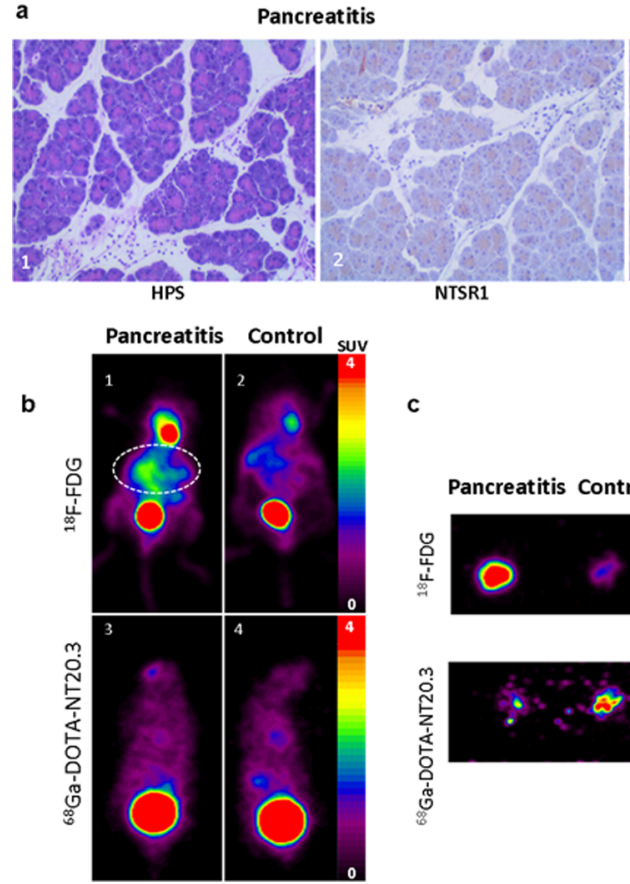

c

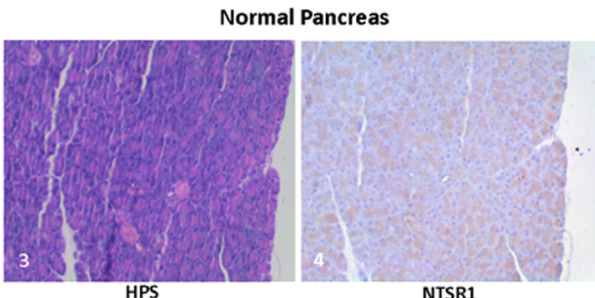

d

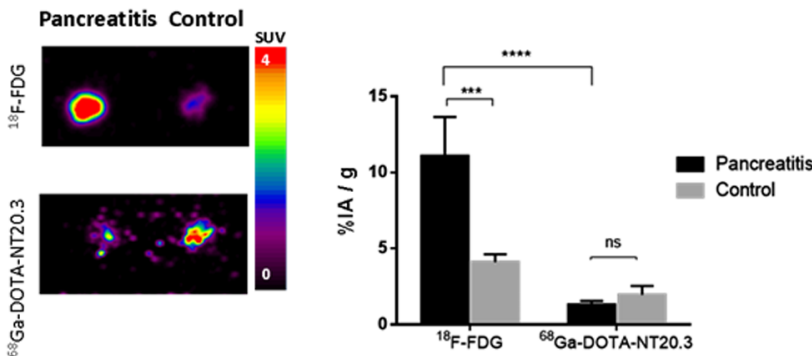

Figure 6. (a) Representative histological sections of mouse pancreas fixed in $10 \%$ neutral-buffered formalin, paraffin embedded, and stained with hematoxylin phloxine saffron from a caerulein-treated one (panel 1) and from a control mouse (panel 3). Original magnification $\times 100$. On adjacent slice NTSR1 labeling was performed (panel 2, 4). Representative in vivo (b) and ex vivo (c) PET images of caerulein-treated mice (left) and control mice (right) with ${ }^{18} \mathrm{~F}-\mathrm{FDG}$ (up) and ${ }^{68} \mathrm{Ga-DOTA}-\mathrm{NT}-20.3$ (down). (d) Uptake of imaging agents in the pancreas of control and treated mice as determined ex vivo by weighing and counting the organs. Data are expressed in mean \% IA/g \pm SD $\left(n=4\right.$ for the ${ }^{68}$ Ga-DOTA-NT-20.3 caerulein-treated group, $\mathrm{n}=3$ for the ${ }^{68} \mathrm{Ga}$-DOTA-NT-20.3 untreated group, $n=4$ for the ${ }^{18} \mathrm{~F}$-FDG caerulein-treated group, $n=3$ for the ${ }^{18} \mathrm{~F}$-FDG untreated group). $* * * P<0.005 ; * * * * P<0.001$.

formation, particularly in case of the two most common precursor lesions, PanINs and IPMNs, that can progress to PDAC. ${ }^{23}$
Specific probes could improve the diagnostic imaging and tumor staging by detecting metastases, recurrence, and could help in monitoring response to therapy. ${ }^{24}$ NTSR1 expression 
by cancer cells triggered the development of many radioactive NTS analogues with a limited number radiolabeled for PET imaging and only in preclinical studies. ${ }^{25}$ Among them, the $\left[{ }^{68} \mathrm{Ga}\right] 8$ derivative (Lys $\left(\mathrm{PEG}_{6}\right.$-NODAGA)-Nlys-Lys-Pro-TyrTle-Leu) showed, in a subcutaneous graft, a moderate tumor uptake of $1.55 \% \mathrm{IA} / \mathrm{g}$ at $1 \mathrm{~h}$ p.i. but a high tumor-to-blood ratio (value of 31 ). Kidney uptake was very high $(45 \% \mathrm{IA} / \mathrm{g}$ ), leading to a poor tumor-to-kidney ratio (value of 0.03 ) at $1 \mathrm{~h}$ p.i. ${ }^{26}$ Recently, a DOTA-conjugated NTSR1 antagonist (3BP227) labeled with indium-111 showed a favorable tumor-tokidney ratio (value of 5) and tumor-to-blood ratio (value of 21) at $6 \mathrm{~h}$ after injection in HT29 tumor-bearing mice but low clearance from blood and poor tumor to background contrast after $3 \mathrm{~h}$ p.i., ${ }^{27}$ which may be a problem with the usual short physical half-life radionuclides used for PET imaging, such as fluorine- 18 or ${ }^{68} \mathrm{Ga}$ (half-lives of 110 and $68 \mathrm{~min}$, respectively). An initial report provides clinical evidence of lutetium-177 $\left({ }^{177} \mathrm{Lu}\right)-3 \mathrm{BP}-227$ as a theranostic agent for patients with PDAC for treatment and therapy monitoring by single-photon emission CT. ${ }^{28}$

In previous works, we further investigated the structural parameters influencing NTSR1-positive tumor uptake of labeled NTS analogues to improve binding affinity and in vivo stability and selected NT-20.3 and its DTPA (diethylenetriamine penta-acetic acid) or DOTA conjugates as the most favorable tracers based on extensive analysis of ${ }^{111}$ In-labeled DOTA-NT-20.3. ${ }^{17}$ Small linear peptides may adopt preferential conformations in solution depending on the nature of the metal in the complex with DOTA that influence the binding to receptors and the hydrolysis by proteolytic enzymes. Unfortunately, the short half-life of ${ }^{68} \mathrm{Ga}$ prevented us from studying its in vivo stability. However, the very similar affinities for the receptor and in vivo tumor uptake observed in this study and in the previous paper for the ${ }^{111}$ In-DOTA and the ${ }^{68} \mathrm{Ga}$-DOTA peptides suggest that their in vivo stability must also be similar.

In this study, dynamic PET imaging with ${ }^{68}$ Ga-DOTA-NT20.3 revealed focal uptake in the subcutaneous and orthotopic grafts of human PDAC AsPC-1 cells and afforded high-quality images with low background in NT organs. Biodistribution studies also showed fast clearance from blood and high tumor uptake $(5.28 \% \mathrm{IA} / \mathrm{g}$ ) leading to a tumor-to-blood ratio value of 6 at $1 \mathrm{~h}$ p.i., and mainly excretion by the kidney with an uptake value of $5.38 \% \mathrm{IA} / \mathrm{g}$, leading to a tumor-to-kidneys ratio value of 1 at $1 \mathrm{~h}$ p.i. The significant decrease of tumor uptake in a blocking study demonstrated the specificity of the tracer.

Differential diagnosis of PDAC, focal mass pancreatitis, or autoimmune pancreatitis is still one of the toughest problems in the management of pancreatic disease. Approximately 4$10 \%$ of the pancreatic resections performed for presumed malignancy revealed non-neoplastic disease on pathologic evaluation. $^{29}$ As a consequence, a disease-specific imaging probe that targets specific molecular abnormalities of the disease, rather than depending only on metabolic changes, would be a desirable probe for tumor detection. This is the reason why we choose to compare ${ }^{68} \mathrm{Ga}$-DOTA-NT-20.3 to the standard ${ }^{18}$ F-FDG PET imaging in an orthotopic xenograft model versus an experimental acute pancreatitis induced by caerulein that is reminiscent of the clinical manifestations of severe acute pancreatitis. ${ }^{19}$ Similar to von Forstner et al. using ${ }^{18} \mathrm{~F}-\mathrm{FDG}$ in PDAC orthotopic tumors ${ }^{30}$ we found a low uptake of ${ }^{18}$ F-FDG in orthotopic AsPC-1 tumors contrasting with the good detection of tumor on PET images with ${ }^{68} \mathrm{Ga}$-DOTA-
NT-20.3 only 14 days after cells' implantation. As reported by Dong et al. we have evaluated the extent and quantification of pancreatic inflammation in mice using small animal ${ }^{18} \mathrm{~F}-\mathrm{FDG}$ $\mathrm{PET}^{31}$ and, as expected, the in situ inflammatory response was visible on ${ }^{18}$ F-FDG PET but not on ${ }^{68}$ Ga-DOTA-NT-20.3. This result was concordant with the histological observation of pancreatic edema, infiltration by inflammatory cells in close contact with acini and fibrosis, but no detection of NTSR1 expression.

High tumor selectivity and low uptake in case of pancreatic inflammation in animal models support the potential for clinical use of ${ }^{68}$ Ga-DOTA-NT-20.3 as a tool to discriminate PDAC from pancreatitis.

In this study, DOTA-NT-20.3 was labeled with ${ }^{68} \mathrm{Ga}$. Labeling was achieved in less than $30 \mathrm{~min}$ with a yield of about 98\%. Recently, Wang et al. developed a fluorine-18-labeled radiotracer based on the same NT-20.3 peptide backbone and obtained similar PET imaging and biodistribution results. Indeed, $\mathrm{Al}^{18} \mathrm{~F}$-NOTA-NT showed a high tumor-to-background contrast in the AsPC-1 model with a tumor-to-muscle ratio of 7.8 at $1 \mathrm{~h}$ p.i. and a tumor uptake of $3.1 \% \mathrm{IA} / \mathrm{g}$ but also in PANC-1 with an ex vivo tumor uptake of $3.5 \% \mathrm{IA} / \mathrm{g}$ at $1 \mathrm{~h}$ p.i. ${ }^{32}$ Interestingly, other authors developed the corresponding theranostic platform targeting NTSR1 by radiolabeling of the DOTA-NT-20.3 analogue with copper-64 $\left({ }^{64} \mathrm{Cu}\right)$ and evaluated its in vivo imaging capabilities in nude mice bearing HT-29 adenocarcinoma, PC3 prostate carcinoma xenografts, or AsPC1 adenocarcinoma. ${ }^{15,33}{ }^{64} \mathrm{Cu}-\mathrm{DOTA}-\mathrm{NT}-20.3^{33}$ or ${ }^{64} \mathrm{Cu}-\mathrm{AmBaSar}-\mathrm{NT}-20.3$ (with a different chelate) ${ }^{15}$ demonstrated prominent tumor uptake with low background in NT organs, except for kidneys $\left(4.28 \% \mathrm{IA} / \mathrm{g}\right.$ at $4 \mathrm{~h}$ p.i of ${ }^{64} \mathrm{Cu}-$ DOTA-NT-20.3), which could be the dose-limiting organ in case of therapy use.

One can speculate from these preclinical results that ${ }^{68} \mathrm{Ga}$ DOTA-NT-20.3 may have two applications for PET in humans. First, it may be used as a diagnostic tool. The fact that inflammatory lesions do not take up ${ }^{68}$ Ga-DOTA-NT-20.3 should result in high specificity. However, the detection rate of PDAC lesions using ${ }^{68}$ Ga-DOTA-NT-20.3 will probably be lower than the $\sim 80 \%$ rate of NTSR1 expression observed in vitro. ${ }^{34}$ In vivo PET-detectable PDAC lesions would probably correspond to those lesions with strongly or moderately (i.e., scoring $>100$ ) positive NTSR1 immunohistochemical staining, that is, to about $56 \%$ of patients' specimens. The added value of ${ }^{68}$ Ga-DOTA-NT-20.3 PET in patients should be its high positive predictive value compared to other PET tracers, in particular FDG. If ${ }^{68}$ Ga-DOTA-NT-20.3 is positive, an equivocal lesion could be characterized as PDAC with a high likelihood; in contrast, PDAC could not be ruled out in case of a ${ }^{68}$ Ga-DOTA-NT-20.3-negative lesion. The second potential application could be the selection of patients with a nonresectable PDAC for internal radiotherapy using a ${ }^{177} \mathrm{Lu}-$ NTSR1 targeting ligand.

These results make DOTA-NT-20.3 a promising probe for imaging and therapy applications of PDAC and other tumors overexpressing this receptor such as breast ${ }^{35}$ nonsmall cell lung, ${ }^{36}$ colon, $^{37}$ or prostate cancers. ${ }^{38}$

\section{CONCLUSIONS}

To conclude, ${ }^{68}$ Ga-DOTA-NT-20.3 has a favorable pharmacokinetics and biodistribution profile, which results in highquality PET images in mice with subcutaneous or orthotopic grafted human pancreatic cancer. Moreover, by contrast to ${ }^{18} \mathrm{~F}$ - 
FDG, ${ }^{68}$ Ga-DOTA-NT-20.3 was able to discriminate PDAC from pancreatitis in mouse models. Thus, ${ }^{68} \mathrm{Ga}-\mathrm{DOTA}-\mathrm{NT}$ 20.3 showed specific binding to NTSR1 of pancreatic cancer cells and provided higher PET detection of adenocarcinoma than ${ }^{18}$ F-FDG. ${ }^{68}$ Ga-DOTA-NT-20.3 seems to be a good candidate for PET imaging of overexpression of NTSR1 on human ductal pancreatic carcinoma and could benefit patients with suspected PDAC, in case of a positive result. Moreover, ${ }^{68}$ Ga-DOTA-NT-20.3 could be used as theranostic agent to define the patient population most likely to benefit from targeted therapy with ${ }^{177}$ Lu-labeled NTSR1 ligands.

\section{ASSOCIATED CONTENT}

\section{S Supporting Information}

The Supporting Information is available free of charge on the ACS Publications website at DOI: 10.1021/acs.molpharmaceut.9b00283.

Preparation and quality control of ${ }^{68} \mathrm{Ga}$-DOTA-NT20.3; radio-HPLC chromatogram;TACs generated by VOI analysis of dynamic PET imaging performed 50 min after ${ }^{68} \mathrm{Ga}$-DOTA-NT-20.3 injection in healthy nu/ nu mice; and summarized data of the ex vivo biodistribution (PDF)

\section{AUTHOR INFORMATION}

\section{Corresponding Author}

*E-mail: Aurelie.prignon@sorbonne-universite.fr. Phone: +33 156017298.

\section{ORCID $\odot$}

Aurélie Prignon: 0000-0002-3963-863X

\section{Author Contributions}

A.P. and A.G.-G. designed the experiments. F.A. and A.G.-G. performed the chemical design of the peptide. A.P. and C.P. performed the radiosynthesis and the animal experiments. D.W., A.C., and P.F. performed the histochemistry experiments. All authors analyzed the data. A.P. wrote the paper. The drafting or critical revision for important intellectual content of the paper was conducted by J.B., P.F., J.-N.T., and A.G.-G. All the authors proofread the paper. All the authors read and approved the final paper.

\section{Notes}

The authors declare no competing financial interest.

IHC on TMA investigation was conducted in accordance with the ethical standards and approved by the Institutional Review Board (IRB) of Paris North Hospitals, Paris 7 University, APHP, no. 12-059. All applicable international, national, and/or institutional guidelines for the care and use of animals were followed.

\section{ACKNOWLEDGMENTS}

The authors gratefully thank Iason for providing the GMP peptide.

\section{ABBREVIATIONS}

PDAC, pancreatic ductal adenocarcinoma; CT, computed tomography; MRI, magnetic resonance imaging; PET, positron-emission tomography; ${ }^{18} \mathrm{~F}-\mathrm{FDG}, 2-\left[{ }^{18} \mathrm{~F}\right]$ fluoro-deoxyglucose; IPMNs, intraductal papillary mucinous neoplasms; NTS, neurotensin; GI, gastrointestinal; NTSR1, neurotensin receptor 1; TMAs, tissue microarrays; TACs, time-activity curves; NT, nontumor; $\mathrm{SUV}_{\max }$ maximal standardized uptake value; VOI, volume of interest; SD, standard deviation; \% IA/ g, percentage of the injected activity/gram of tissue; p.i., post injection

\section{REFERENCES}

(1) Hidalgo, M.; Cascinu, S.; Kleeff, J.; Labianca, R.; Löhr, J.-M.; Neoptolemos, J.; et al. Addressing the challenges of pancreatic cancer: future directions for improving outcomes. Pancreatology 2015, 15, 818.

(2) Holzapfel, K.; Reiser-Erkan, C.; Fingerle, A. A.; Erkan, M.; Eiber, M. J.; Rummeny, E. J.; et al. Comparison of diffusion-weighted MR imaging and multidetector-row $\mathrm{CT}$ in the detection of liver metastases in patients operated for pancreatic cancer. Abdom. Imag. 2011, 36, 179-184.

(3) Parsons, C. M.; Sutcliffe, J. L.; Bold, R. J. Preoperative evaluation of pancreatic adenocarcinoma. J. Hepato-Biliary-Pancreatic Surg. 2008, $15,429-435$.

(4) Zamboni, G.; Hirabayashi, K.; Castelli, P.; Lennon, A. M. Precancerous lesions of the pancreas. Best Pract. Res., Clin. Gastroenterol. 2013, 27, 299-322.

(5) Pakzad, F.; Groves, A. M.; Ell, P. J. The role of positron emission tomography in the management of pancreatic cancer. Semin. Nucl. Med. 2006, 36, 248-256.

(6) Saito, M.; Ishihara, T.; Tada, M.; Tsuyuguchi, T.; Mikata, R.; Sakai, Y.; et al. Use of F-18 fluorodeoxyglucose positron emission tomography with dual-phase imaging to identify intraductal papillary mucinous neoplasm. Clin. Gastroenterol. Hepatol. 2013, 11, 181-186.

(7) Santhosh, S.; Mittal, B. R.; Bhasin, D.; Srinivasan, R.; Rana, S.; Das, A.; et al. Role of $18 \mathrm{~F}$-fluorodeoxyglucose positron emission tomography/computed tomography in the characterization of pancreatic masses: Experience from tropics. J. Gastroenterol. Hepatol. 2013, 28, 255-261.

(8) Cornelissen, B.; Knight, J. C.; Mukherjee, S.; Evangelista, L.; Xavier, C.; Caobelli, F.; et al. Translational molecular imaging in exocrine pancreatic cancer. Eur. J. Nucl. Med. Mol. Imaging 2018, 45, $2442-2455$.

(9) Vincent, J.-P.; Mazella, J.; Kitabgi, P. Neurotensin and neurotensin receptors. Trends Pharmacol. Sci. 1999, 20, 302-309.

(10) Béraud-Dufour, S.; Abderrahmani, A.; Noel, J.; Brau, F.; Waeber, G.; Mazella, J.; et al. Neurotensin is a regulator of insulin secretion in pancreatic beta-cells. Int. J. Biochem. Cell Biol. 2010, 42, $1681-1688$.

(11) Wang, L.; Friess, H.; Zhu, Z.; Graber, H.; Zimmermann, A.; Korc, M.; et al. Neurotensin receptor-1 mRNA analysis in normal pancreas and pancreatic disease. Clin. Cancer Res. 2000, 6, 566-571.

(12) Dupouy, S.; Mourra, N.; Doan, V. K.; Gompel, A.; Alifano, M.; Forgez, $\mathrm{P}$. The potential use of the neurotensin high affinity receptor 1 as a biomarker for cancer progression and as a component of personalized medicine in selective cancers. Biochimie 2011, 93, 13691378.

(13) Wu, Z.; Martinez-Fong, D.; Trédaniel, J.; Forgez, P. Neurotensin and its high affinity receptor 1 as a potential pharmacological target in cancer therapy. Front. Endocrinol. 2013, 3, 184.

(14) Reubi, J. C.; Waser, B.; Friess, H.; Buchler, M.; Laissue, J. Neurotensin receptors: a new marker for human ductal pancreatic adenocarcinoma. Gut 1998, 42, 546-550.

(15) Yin, X.; Wang, M.; Wang, H.; Deng, H.; He, T.; Tan, Y.; et al. Evaluation of neurotensin receptor 1 as a potential imaging target in pancreatic ductal adenocarcinoma. Amino Acids 2017, 49, 13251335.

(16) Alshoukr, F.; Rosant, C.; Maes, V.; Abdelhak, J.; Raguin, O.; Burg, S.; et al. Novel neurotensin analogues for radioisotope targeting to neurotensin receptor-positive tumors. Bioconjugate Chem. 2009, 20, 1602-1610.

(17) Alshoukr, F.; Prignon, A.; Brans, L.; Jallane, A.; Mendes, S.; Talbot, J.-N.; et al. Novel DOTA-neurotensin analogues for 111In 
scintigraphy and $68 \mathrm{Ga}$ PET imaging of neurotensin receptor-positive tumors. Bioconjugate Chem. 2011, 22, 1374-1385.

(18) Hoffman, R. M. Orthotopic Metastatic Mouse Models for Anticancer Drug Discovery and Evaluation: a Bridge to the Clinic. Invest. New Drugs 1999, 17, 343-360.

(19) Lerch, M. M.; Gorelick, F. S. Models of Acute and Chronic Pancreatitis. Gastroenterology 2013, 144, 1180-1193.

(20) Lerch, M. M.; Gorelick, F. S. Models of acute and chronic pancreatitis. Gastroenterology 2013, 144, 1180-1193.

(21) Körner, M.; Waser, B.; Strobel, O.; Buchler, M.; Reubi, J. C. Neurotensin receptors in pancreatic ductal carcinomas. EJNMMI Res. 2015, 5, 17.

(22) Wang, J.-G.; Li, N.-N.; Li, H.-N.; Cui, L.; Wang, P. Pancreatic cancer bears overexpression of neurotensin and neurotensin receptor subtype-1 and SR 48692 counteracts neurotensin induced cell proliferation in human pancreatic ductal carcinoma cell line PANC1. Neuropeptides 2011, 45, 151-156.

(23) Distler, M.; Aust, D.; Weitz, J.; Pilarsky, C.; Grutzmann, R. Precursor lesions for sporadic pancreatic cancer: PanIN, IPMN, and MCN. BioMed Res. Int. 2014, 2014, 474905.

(24) Ansari, D.; Gustafsson, A.; Andersson, R. Update on the management of pancreatic cancer: Surgery is not enough. World J. Gastroenterol. 2015, 21, 3157-3165.

(25) Maschauer, S.; Prante, O. Radiopharmaceuticals for imaging and endoradiotherapy of neurotensin receptor-positive tumors. $J$. Labelled Compd. Radiopharm. 2018, 61, 309-325.

(26) Maschauer, S.; Einsiedel, J.; Hübner, H.; Gmeiner, P.; Prante, O. 18F- and 68Ga-Labeled Neurotensin Peptides for PET Imaging of Neurotensin Receptor 1. J. Med. Chem. 2016, 59, 6480-6492.

(27) Schulz, J.; Rohracker, M.; Stiebler, M.; Goldschmidt, J.; Grosser, O. S.; Osterkamp, F.; et al. Comparative Evaluation of the Biodistribution Profiles of a Series of Nonpeptidic Neurotensin Receptor-1 Antagonists Reveals a Promising Candidate for Theranostic Applications. J. Nucl. Med. 2016, 57, 1120-1123.

(28) Baum, R. P.; Singh, A.; Schuchardt, C.; Kulkarni, H. R.; Klette, I.; Wiessalla, S.; et al. 177Lu-3BP-227 for Neurotensin Receptor 1Targeted Therapy of Metastatic Pancreatic Adenocarcinoma: First Clinical Results. J. Nucl. Med. 2018, 59, 809-814.

(29) Abraham, S. C.; Wilentz, R. E.; Yeo, C. J.; Sohn, T. A.; Cameron, J. L.; Boitnott, J. K.; et al. Pancreaticoduodenectomy (Whipple resections) in patients without malignancy: are they all "chronic pancreatitis"? Am. J. Surg. Pathol. 2003, 27, 110-120.

(30) von Forstner, C.; Egberts, J.-H.; Ammerpohl, O.; Niedzielska, D.; Buchert, R.; Mikecz, P.; et al. Gene Expression Patterns and Tumor Uptake of 18F-FDG, 18F-FLT, and 18F-FEC in PET/MRI of an Orthotopic Mouse Xenotransplantation Model of Pancreatic Cancer. J. Nucl. Med. 2008, 49, 1362-1370.

(31) Dong, X.; Zhan, Y. C.; Jiang, R. Y.; Xie, Q. P.; Peng, J. P.; Wang, J.; et al. The Potential Effect of Proteasome Inhibitor PS-341 on Severe Acute Pancreatitis Detected by Positron Emission Tomography in ICR Mice. J. Surg. Res. 2010, 162, 193-202.

(32) Wang, M.; Zhang, H.; Wang, H.; Feng, H.; Deng, H.; Wu, Z.; et al. Development of [(18)F]AlF-NOTA-NT as PET Agents of Neurotensin Receptor-1 Positive Pancreatic Cancer. Mol. Pharmaceutics 2018, 15, 3093-3100.

(33) Deng, H.; Wang, H.; Zhang, H.; Wang, M.; Giglio, B.; Ma, X.; et al. Imaging Neurotensin Receptor in Prostate Cancer With $64 \mathrm{Cu}-$ Labeled Neurotensin Analogs. Mol. Imaging 2017, 16, 1536012117711369.

(34) Buchegger, F.; Bonvin, F.; Kosinski, M.; Schaffland, A. O.; Prior, J.; Reubi, J. C.; et al. Radiolabeled neurotensin analog, 99mTcNT-XI, evaluated in ductal pancreatic adenocarcinoma patients. J. Nucl. Med. 2003, 44, 1649-1654.

(35) Souazé, F.; Dupouy, S.; Viardot-Foucault, V.; Bruyneel, E.; Attoub, S.; Gespach, C.; et al. Expression of neurotensin and NT1 receptor in human breast cancer: a potential role in tumor progression. Cancer Res. 2006, 66, 6243-6249.

(36) Alifano, M.; Souaze, F.; Dupouy, S.; Camilleri-Broet, S.; Younes, M.; Ahmed-Zaid, S.-M.; et al. Neurotensin receptor 1 determines the outcome of non-small cell lung cancer. Clin. Cancer Res. 2010, 16, 4401-4410.

(37) Chao, C.; Tallman, M. L.; Ives, K. L.; Townsend, C. M., Jr.; Hellmich, M. R. Gastrointestinal Hormone Receptors in Primary Human Colorectal Carcinomas1. J. Surg. Res. 2005, 129, 313-321.

(38) Swift, S. L.; Burns, J. E.; Maitland, N. J. Altered expression of neurotensin receptors is associated with the differentiation state of prostate cancer. Cancer Res. 2010, 70, 347-356. 